\title{
PARACOCCIDIOIDOMICOSIS PULMONAR
}

\author{
Bres. LEOROLDO ARCOS V. y JOHGL RORHESUF?, C \\ Departumenta Wéfico del Segrero, Quifo
}

Is parabuecidjoidomicosis, Jlamida lambirn blastomicosis suchanericana " enfermedad de Luiz, es una enfurosdad enderrica en nuestro país. En su localizición pulnesnár se confunde frecuentemente con otrus afecciones, particularmente con la thlerculosis. Fil escudio mieclógìco no es siempre J'ícil pará nusotros por la falta dis laboratorios especializados. Por otro ladu: tiene un iratanininto especifitico rjue es sclativamente sencillo, poro cosiosus $y$ de excelentes rosultados. For las $x$ tzorues indicadas, has historias clitricas an sue se detalla las manifestaciones, Io evolución de la enfermedad y el trasumiento realizaldo $a n$ cuatro rasos, pueden ser die interús par los médicos. Fstus asos fteoron adtritidus en el Dixpentsátio N' 1 de LEA de Quito ent'e agosto de 1962 y junio de 1964 .

CASO N" 1. J. T. P. (IIistoria 14127). Agricultur do 38 años de cdad, examinado el $14 \mathrm{de}$ agosto de 1962 . Nacio y ha residido en Cha. huspamba, zona trupical de la Pru. rincia tle Loyja, La enfermedad actual comenzó en febrero de 1962 enn sensilcól sle estoubuen el lado izquiereln de la faringe ryue ne hizo más notable desde mayo quo flue cuando se notó aumento de volumes en la urrigrlala izgutie rda. La molestiv, sexpmpana da de wornezón, descemdió por la parte iutíc ror de encllo thasta la región rotroesitrnal. En las filtimas semanas hubir también algo de dolor en las zomas unovertebrales, además de estorbo y dolot $\mathrm{co}$ la amígilala derecina. Ha presentado ulceruriones en la encia inicrior por lo cual le extrajeron las pocan piczas dentarias que lo quedalum, sin resultado nobre las veleridas Iesiones. No ha habido fiebre durante todo at transcurso da: la enfermedad: poro ba tenicto anorexia y perdicla de peso. Fin cl examer fisico se encontró al pacion.

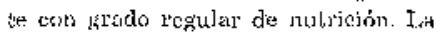
amígrlola izquierda estiaba grande, prominesile, At unos $3 \mathrm{~cm}$. fle diámetro. con superticio de nspecto géanuloso, rojo intenso y con tendencia a sumgris, de consiriencia suave y dolorosa. $\mathrm{L}$ a 
amigdala derecha tambjén eslatbi alumeniatiz de volumen, de color doigizos y

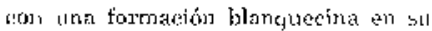
polo irferion, Uluckis jequeñas so veitu en el borde del maxilar inferiris:

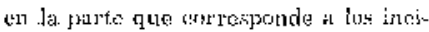
sivos medius. Los pilines, ]a úvula $\mathrm{y} \mathrm{rl}$ resto de la mucosa bucul toníun aspec.

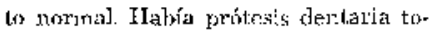
tivl. Los pulmomes eran claros a la percusion y se auscultaba illumdantes es:tertores húmodur diseminates o: au" bos ladus. :Fl resto del examen físicu ner hal ló datos importantes. La tensión ar-

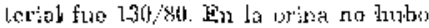
nate anormal lo ıismo gutac on la sangíe, excepto eritrosedirrcntación de 22 mra. Se wifueluć una biopsia do la amíg-

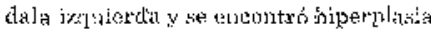
seudocpiteliomatusa reaccional, granu-

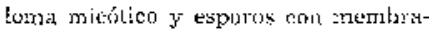
na birrofrumente $y$ protoplasma porsitivo al $\mathrm{PAS}$ (Fi.s. 1) (Dr. Gate Hidalgo), No se pudo continuar ]a investignción prráguc el parsiente regresó al lugar de residentia. Se comunicó ol hatlazgo a un núrlino die Jaja quicn el 6 de septicbre de $1 \$ 62$ practicó amigdaletomía jzquierda.

Regresó el 18 ale enero de 196.4 y refirió yue a la sintonatología descrita anteriormente se hobía sumado tos progresiva m3as imtersen $y$ experturación amauillenta y verdosa con estrías de sangre. Uma vez hubo hemoptisis de méts o menus 80 ec. 1)cste agosto de $1 \$ 92$ lambión labiut presentado repetidas epistaxis que se hicieron mús freuzentes en los últimos dias y deste djrierbre empezó a notar enronqueciruirento de la voz. Como medicación, a

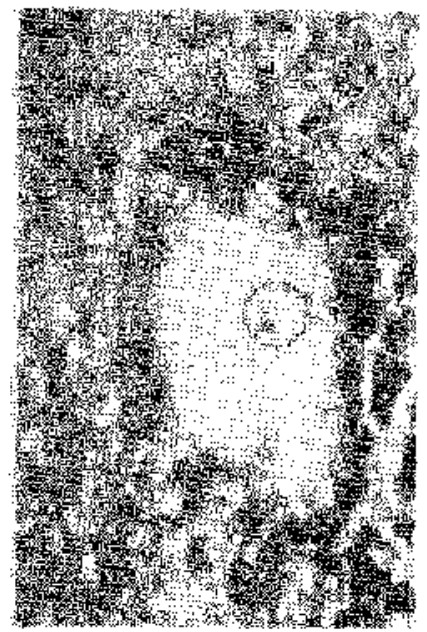

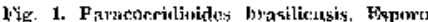
colz inembran birrefrimisende.

raí de la amigdalectnmía, recibirí por seis selnanas griseofulvina, nistatina y otros dilerentos antibióticos sin efueto sobre la enformeded. Fin exploración fúsicat se emeontrí varials pequeñas u1ceruciunes planas de lonrlo gaxis er la

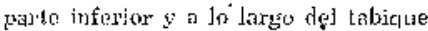

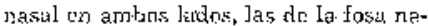
sal derecha con restos do sungre. Habia inflamación y tres ukeracioncs en la emcia inferion y una ulceración en In encia supcrior: Yuals úlceras aparectam co:) bozdes inflamados y fomelo gr:súroo. La amígrtala jzquierda cstrtbán ausente y la derecha hipertrofiada y con dos úíceras poçueñus; dolí: al presionstrla. Bajo el ángulu maxilar derechos 
ar palpaba un ganglio de 2,5 pisy 1,4 aı. slum $y$ medianamerte sersible. En

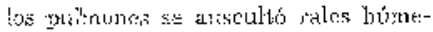
des: pequeñfus y mediaros suprit e in. Fritiaviculars a 2 ctilerdos, ed restím esajiolovercelsal derecha y busc postc-

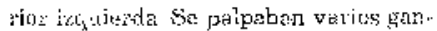
slius do hatitir ur centínetro Jigere"rente crusibles en ambas axi?as $y$

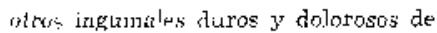

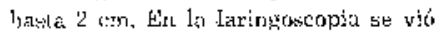

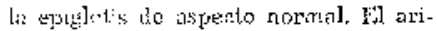
teroties derecho are de vuluine: do 3 o 4. vecess de lo que aparecít el irquiesdo nommal. Fin la cara montorn:

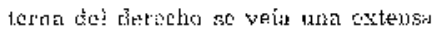

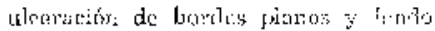
siris que se extendía his:lis la cumisu-

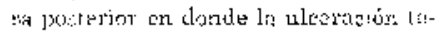

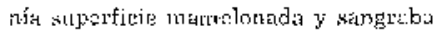
‘un favijuat. Cherdas vocaíos supuriores y verdatrata normales en el lis-

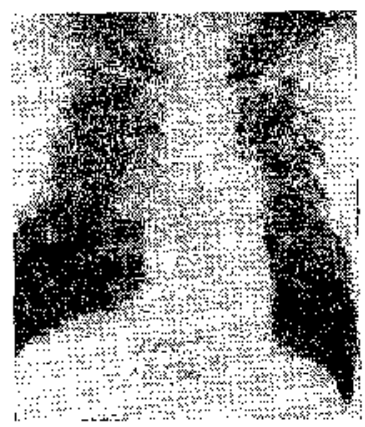

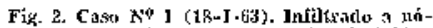
dulos de merisano tartanio mat defivirtos, cun'ltentes, resiputintdo las hases. Hilius fuertementc engrosiadas.

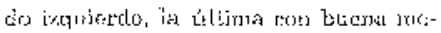

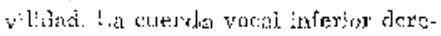

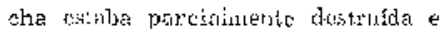
in (ii) jzquierdio ste jotabeis titcorasionss

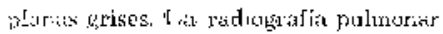
de: 1 \& do de nóntulos de ucdimo tanaño mal

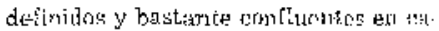

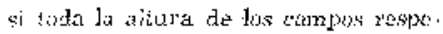

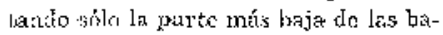

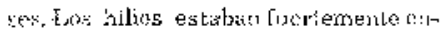
gremades. Titua place radiográlica de cincu meses atrís presentaba nitera-

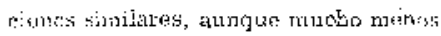

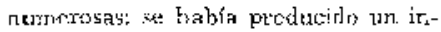
eremertu de cenfo pot sientes on in in-

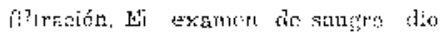

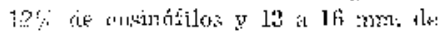
exitronedimentixtión tono datos anor-

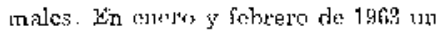
cspute un microscópiso fue neǵaiivu pata bacterias ácidn-resistentes y twis cultivos negativos para bacilo tubereuloso. Tos resultados de varios exámenes micolóstions electuados extrn 21-L y 25-IT-fi3 fierm: Un asputo y pias de ungédala neirelivos en examen dirncic.

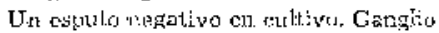
ax!lar mogat:vo en cultivo. Un esputo negrative en inosulación. Jos Asputos y un lavado bronquial. prisitivos pard bontugos ro idendititendos (C. immitis?, H. (mpsulatum?) on cultivo. Lin esputo positive para $\mathbf{P}$. brasiliemsis en jootudacén al cobayo. Biopsia de melcosa gingivol: gingivitis subaguda intonsa, no granuloma, no hongos. Biopsial de laringe: granuloma inflamatorio con células gignantes, hiperplasia seudo - epileliornatosa, no organismos. 


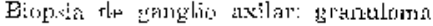

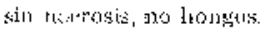

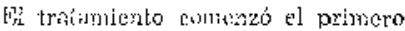

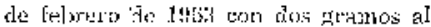
dis: de \{riple sulfe. (Di:umczou! Lión). Al cabo kla ti3 dias thates lus síntomas

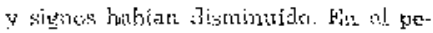
cho $y$ in los ancebrazs apureció une

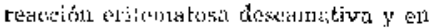

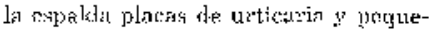

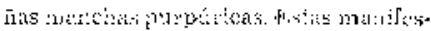

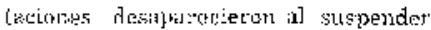

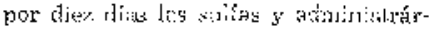

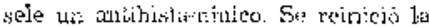
mexdicactón ton I rịle sulfá y ise "mantu."

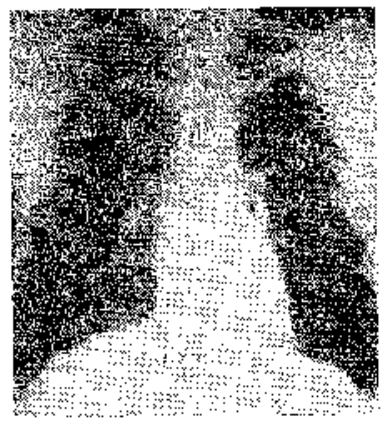

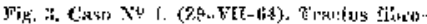

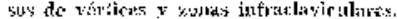

to hasta cl 9 de abril ( 6 semanatis en ity tal), tiompe al cabo det cual tenía solamerste tos ockisional con escasa expactora: ión y ningura lesión de da nariz, boca, fiwinge, ni laringe. So le prescribió 2 dosis diarias de 500 mili-

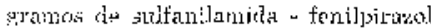

(Orisul Ciba) quo recibis por 15 sema-

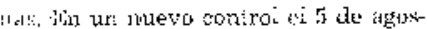
io rle 196:3, ro presentaba ningén sinoma pulmonax, ni había lesiones musosas. In ultima ocasión que se le vio

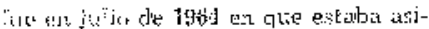
mismo asintomático; nutu, sin tubarso, había asomado ura pequeña ulorración en 4 surco jingivolabial infexior Se le inulicá que tottiara sulfame lox:piridacina. En radiografía pulmo-

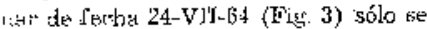
ubservidhan tractoo librosos en vórtices $y$ veriones infraclaviculares, más on el r.

\section{RESUMIEN}

Agricultor que vivía en región tropickl rie la Provirn?a de Loja, de 38 aïos de edact. Por seis inescs aciasaba molestials de la boba ocisionadas por arvigchalinj; y gingivitis ulearada. Posterismente i] mucosn bucal se uleeró unxis exlexntiilitente y so presentaron alrericiones sinilares en la nariz, en la lizringe, en la tráquea $y$ d'n yamglios su-

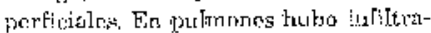
"isll rodulat conflueste bilateral y posibls aderiitis hhiliar. Fxámenes bacten riológicos florom nogativos pura tiberculosis. Fn biopsia de anigchala y esputo se idcntificó ol Paracoccidioides brar

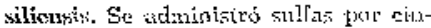
no moses y se abtuw curación de la afección de Jas rucosas, ganglios $y$ pulrúb, Sin emlsargo, cevé de un año despućs se presentó nueva ulceración gingival. 
C.SBO N 2. A. H. V. (Historia 14689).

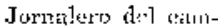

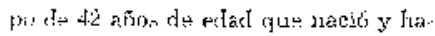

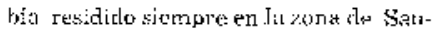
to Doniryo de las Colorados $y$ tenia al mornento su Sabitación en el Kriló-

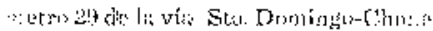
ne. Covsultó e] ity-LII-63 polque derdo aproximatamente dos meses $y$ me-

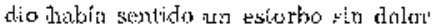
en li parte disjecha posterior de la bnsa a coníacto son los alimentos. Negó glate luviora tas, expecturacitio ni otros

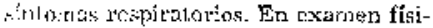

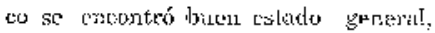

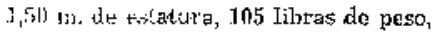
72 : de pulso, 37,3 C de temprreturin $y$ $120 / \mathrm{S}$ ! de presión arterial. Fin la boca,

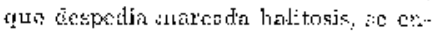
contris In dictadura muy desasenda $y$ era ustarto de jnitensa y exfensa periodontitis. Habia una ulceración de 6 por 2 wn. que isu deside el extremo pustetit:r de las encias $y$ de pilat anterior del velo del paladar hacia adelante $y$ adentto basta cerca de Ia linea medio en ol paladal duro en el lado dexecho. I ic lesion era de bordes planos, do for.. do grisíteco ron algo de tejiơ de piranulición. Otra uleeración ils aspecto semejo:nte se veía en la encía superior izvuiurdu, inmediatamente por oncima de lazi yiezus dentarias $y$ modía 5 pov'] cm. li] resto del examen físico

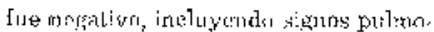
tiartes, En radingrafia pulmonar de 26 JiT-63 (Fig. 4) se apreciatha infiltración exudativa entre el $3^{\circ}$ y $5^{\circ}$ amos sosiales anteriores izqujerdos con una o más excrvaciches irregulares e infiltrato bronconcinoso de la base drre-

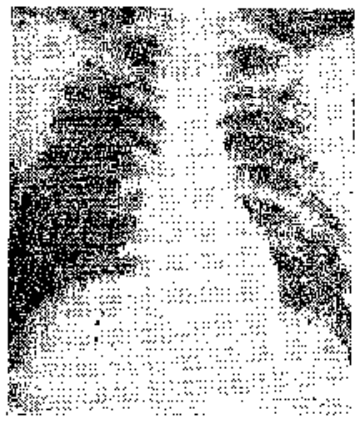

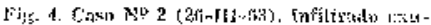

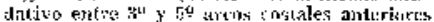

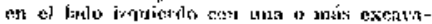

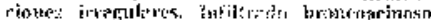

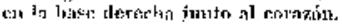

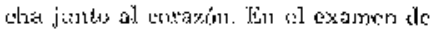

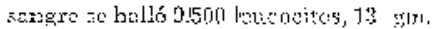

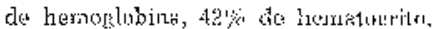

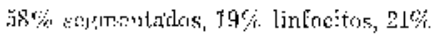

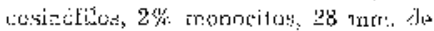
or:zoyetimentacion on teme hora. Fer In oring huldo vestigios de albúmines y

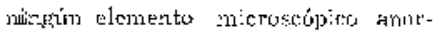

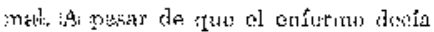
:o ience tios ni expentorasión, por lovar

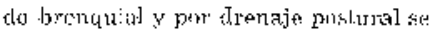

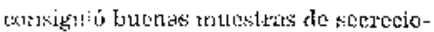
jos fe aspecto muco-puxulento hemup-

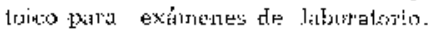

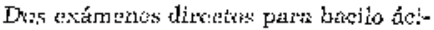
dri-resistente fuerou negativos. I'yeron

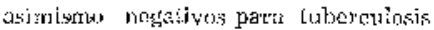
i.res al]livas y dom inoculaciones al cobayo. Ea ux examen th liesco do se excontlon buevos rle paraginimas. Un cultivo de esputo realizado en los Laboratorios $I d]=2$ dio como resultado el 


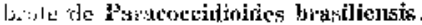

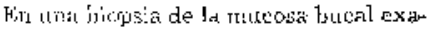

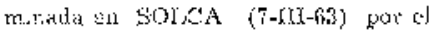

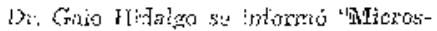
acoico: Sire iratid de porcions:s; de mu-

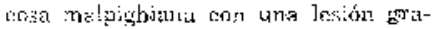

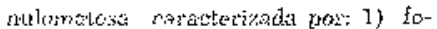

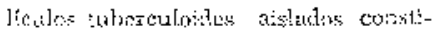
iulílos pö célul:as gigantes, cólulets ep:-

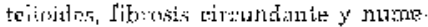
röios linfocitos; es) jntiltraicion dffura

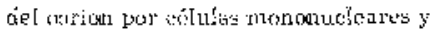

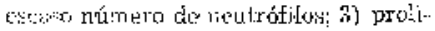
Seración endotelial y capilar; 4) nume ross microaldecesos dentun del epitelio y rácubjér una inliteración difusa tol mismo por prolinucieures. Tactis on Tos joliculos titbroulotues cons en to:s inirroubsesos se thacrul nameruas ostumlas de tpo blastomicoss PAS

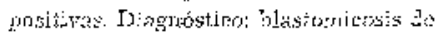
la rrtucosa bre'sl".

绍e instituyó tratamisto con dos do-

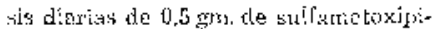
ridacitia-su!fane1aciona (Diamezol Lonto Liff) rlurante 120 días y con unil clo-

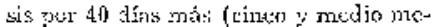

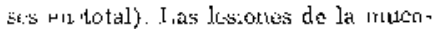

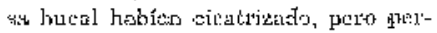

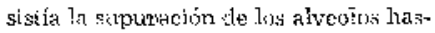
(a roviershre de 1963. (EL pacionte se bjok la cxtrascićn deritarja crue $k c$

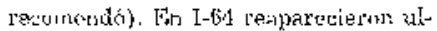
ceracious husta ciess y ahora dumbín ex los narrides. Sin coribsige, jor madiografí (7-I-fik).

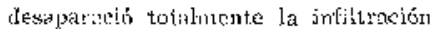
de: lato derecho y casi edrrypletamentic

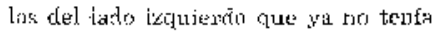
'avidedes. Sr deinicio el tratamierto col ta misina combinación de stalfas, perro el particnte loní $r$ medicuntento sós intils poens dias, reurresendu en ingusto do 1966 en cilue st encuncró el-

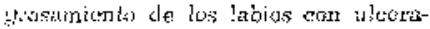

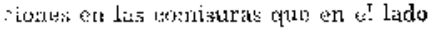

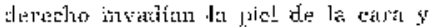
t.,itahon unthertas do zosl.ras sobru un

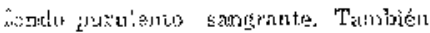
ses concotréró numbrosns ujeeratemes

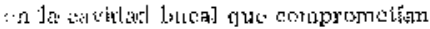

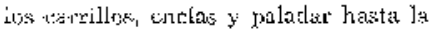
iviula y Ioss pilaress anteriores y las arighalas. Hstas lesince sangrabun con facilidiad y no arar detorosas. La :aliugesiĺfi pulmuna: en esta urasión (25-Vibl-f6) Tulisstró reantivarión de las

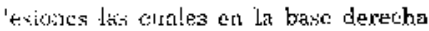
cren mús extransas que orginalmente dos ăthos y unetilo at tás y en el izquięr. the veriatn de mulvo extiavajemes.

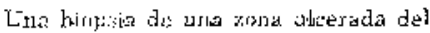
libi, en examen microscópico (31V!II. Gis dio tome resultado grasulo-

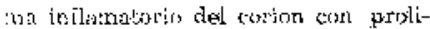
Perrución: crnjuluntivia, colecciones de ]infocito: y algunas sćlulas gigintes inul-

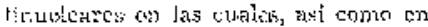
in linfucitos, se ofservalua esférulas lyorthingences sin ytirias iatelales que ¿urun cusideratas como de probables 7". husiliensis.

So volvio a ver al orlernzo el 17-X67 con muy blex extado gencred. Por sul cedeuta thatbra tom mado sialtas de ma-

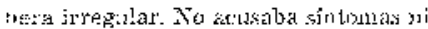
ismes pilmoneres y la rattogrâtin

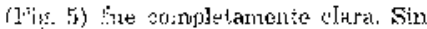

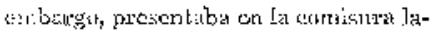
bial' iralierta ana b́ltera vegelante de 5 хаты. у ел la centá inferna de los carrilos y encias steperior e inforior extem- 


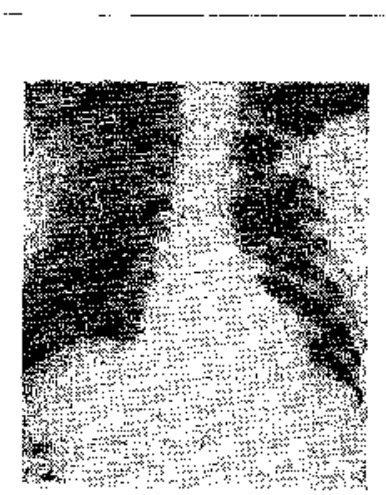

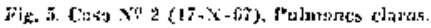

sas pórdidas dis slsianceia de bordes ne-

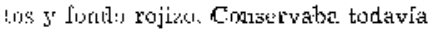

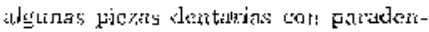

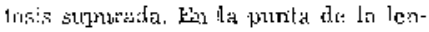
tylu, en ol boude deresho $y$ en su tara Ixiterirys se obserwaban varias fry'mal-

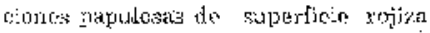
graulusa'y periteriu de coles gris

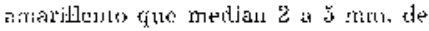
rljámetescs.

\section{TEWUTHEX}

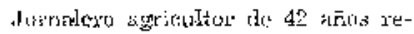
siricule en Iá \%orid de Santı, Domingtu de tur Colorusis con síntomez buxales pis! dos mescs y tuendiu y zin manifest:i-

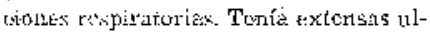

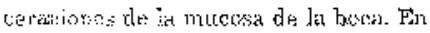
1alicgrafia pultwonar hubo lesiones bi-

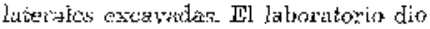
rosultarhe angativo para tuberculosis $\mathrm{y}$ cin exputio y biupsias de la mucose bu-

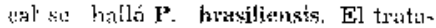

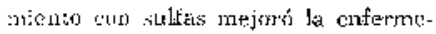

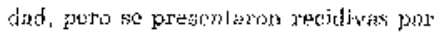
tratemiento irregu'al $y$ discontinua. A.' źli.jnes se vio que las lesiones mul:osils sllifieron sotable incremitnto e invadieron lis piel, mismives las del prulIIón halıian desajarceido. Pnsjbienerk

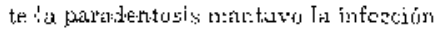

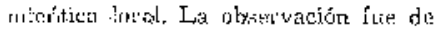
ciletur Hros $y$ mediu.

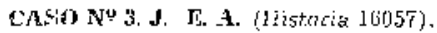
Adiritido el 17 .

VI.6p. Saste de th años de cdad, nal

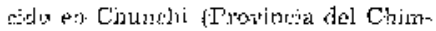

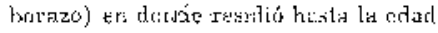
de 90 :

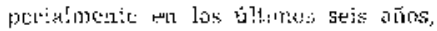

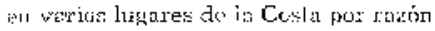
de : u crnpleo en el Ejfrusío. En diciem-

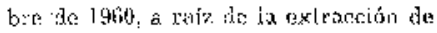

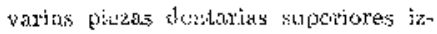

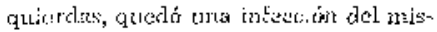
mo siliu, que ss descattó por radiogran fía que fiere debida a proceso alveoliz,

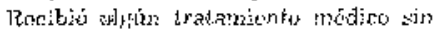

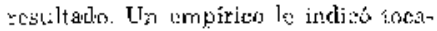

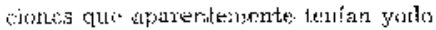

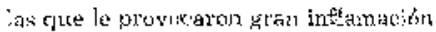
ǵngival pur 'o clael. ingresó al Howpital de "Cuence donde se ruljzó biopsia $y$

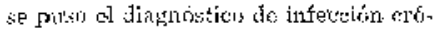
1jon. ligresó exmburticio viso al rlospita:- Xt:iital de Quito y ac conrobobó a: encllo y por radiografía inf $y$ ltrata pulnesran, pur lo que fre translcrido al Seun aturio de LEA de Riobamba. T] estado general cera maio, tenía astenia 
I' anorexia y se cneontsó bistijosenpia posiliva (ácido-resicientes en exasa cantidad) eat dos exúnerdes un marzo y

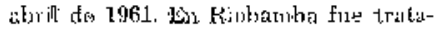
dir con isoniacide y eatreptun icina por seis meses (xle marzo a sieptichabra rite 19tid) pero se obserso que dee aturi: a

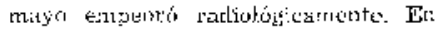
srptiember der 1961 escresó meiciada. pires despures voivib otra vez a. pro.

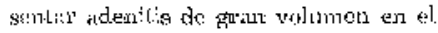

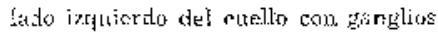
diutis, móviles, thisorosos y uur supa-

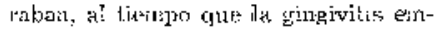

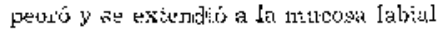
$y$ palácina. Acultió a SOT CA de Gua-

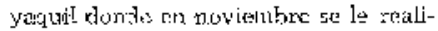

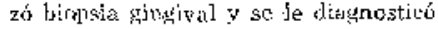
f.lastominomic sudamevireana $y$ ex: radio-

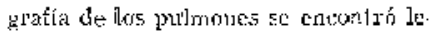

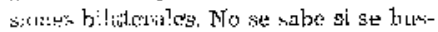
có minosisis pulmonar. En al Ifospital Mitilur de la mïsrth ciudud aeenió 500

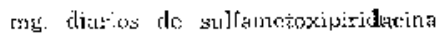
durante un yes y mojoró apreciablemenlu. A prinr:pios de 1062 se presenLis hemoglisis de unos $250 \mathrm{~cm}$. po: lo

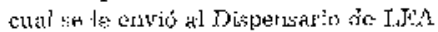
"Ciulos Julio Arosiemena". Aquí lâ ra-

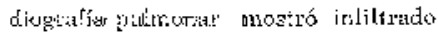
excavarlo parascarctínco derecho e infilo

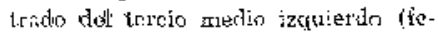
brero de 1962) y entre febrem y mayr de? fi2 luubo siow bacidoscopias negatim vas $y$ un cultivo regativo pat a bacilo tubesculazc, rsciliendo extreplomivina

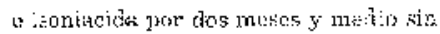

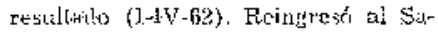
vatorio de Rivbamba en al clue pertnanexió dla jumio de 1962 a eneru de 1963 $y$ on doude cuatro unicruscúpicos y

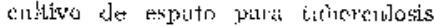
fuertor negrativos. No trvo lartamiento

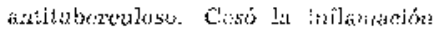

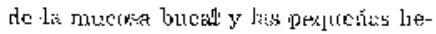

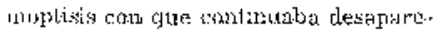

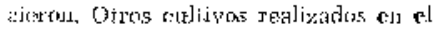

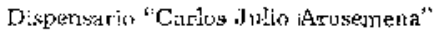
arili te abril de 19fi2 y melyo de 1963 (tialo do expouto y tres contenidos gástricos) fineron de nurvo negutivos para bra: ch novientre del risumo año, luess do :12ix mrarcha formata $y$ de un enfriamicrito busuco, le aprilición ronquera

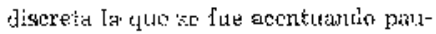
latindrunnto birisla haxerse afonla casi crinpleta. Corn este nicyo síntoma irl-

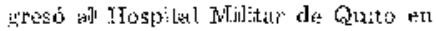

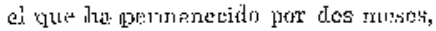

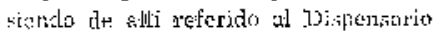
$\mathrm{N}$ ? 1 de Lea u' 17 de juyio de li3ft.

Fiy of exa:nen del' paciende se encon1.ribu ro:жuge appeciable, casi alonía.

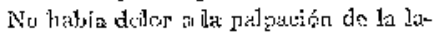

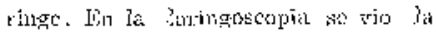
cucerla vacrel jzirgiterda ongrosarta, c11briceitu a là cuecd voual inferior, ulcorudic $y$ tom formasiones mamelonada: que prytucinn tiexto grado de cste. nos:s que se niatjifistibba.por un cornaje ste mediana intemitirad. Se efentuó bjopsia del tojjdo atuosmal pasu exa-

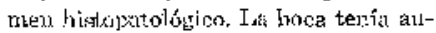
areneia total de piezas dentarias rem. pIaza, तas jor prót ssis. Ni en bocel ni en faringe hubo formatión alguna uleom rativis ot rle granualeión peru el surco cringitivo-labial] superiors irgquiewdo en zus dais tercios atutcriores, se apreciaha reducido en siz profundidad como si subiera sido sitio de una extensa ulce- 


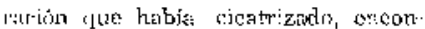
l.tulitre, sin etrbargo, I. m micosa de :injlecto normal. La tomografica de la

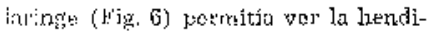
Iura glótsca esterchar $y$ desiplazada hatria la de:cetha, ins cuerdas vonales iz.

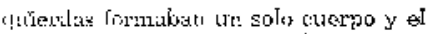

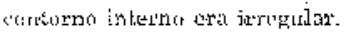

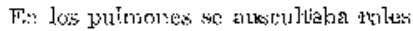

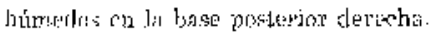

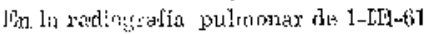

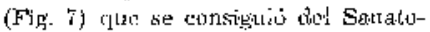
liu de Riobiis?lu se vem lesione:i exu"

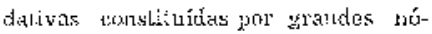

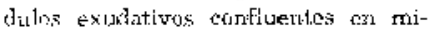
sact inletior terersetea y tercio medio iz-

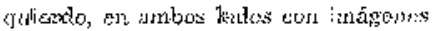
isregultares do atcecteveión.

fit resultates de ia biopsiat da lan'iti.

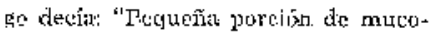

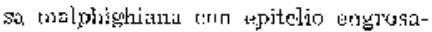

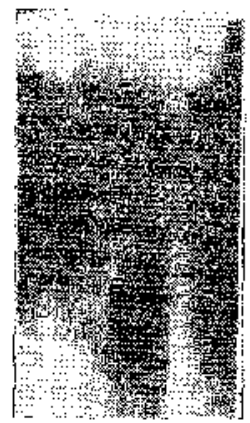

Fis. 6. Cono X0 : Tornugraila fe laringe, Hen.

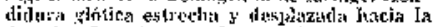

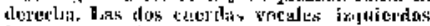

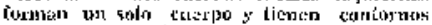

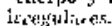

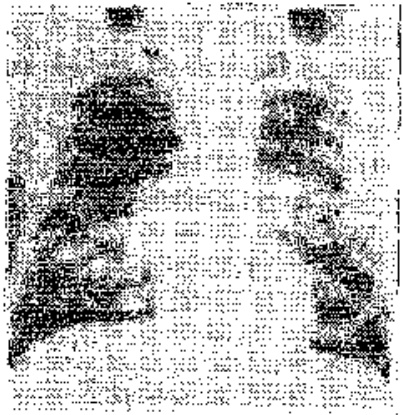

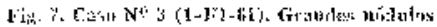

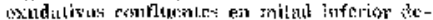

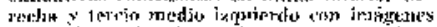

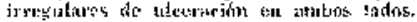

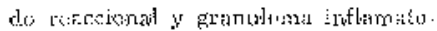

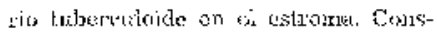

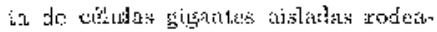

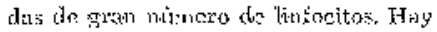

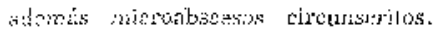

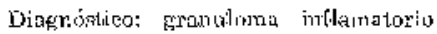
(probable micosis)" (Dr. Gulo Ijrla]go). Cultivn ácI aspute clio desarmollo de Cendita sp. linn tuicrascópyito no se oneoralró baceilo scido-resistente; asi-

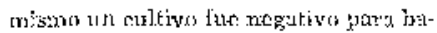

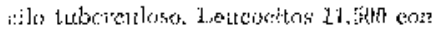

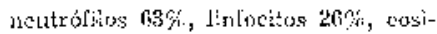

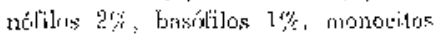
$5 \%$, hematcerito $43 \%$, hemogrtobinal 1.1,6 sill, tritrusedimentavion 27 mill.

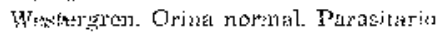
de heces: ruvistes de ancha bistolitica.

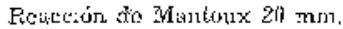

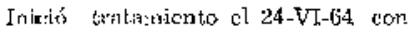
ir:ple sutfa co dosis de $4 \mathrm{gm}$. al diti $\mathrm{pm}$ unrat se? 


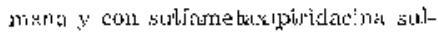

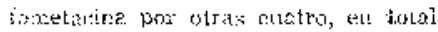
b. en jallingostopia se veía todelvia inlilLac:ón de lia bande veritriculdar $y$ de ir. cuerta vos: vard

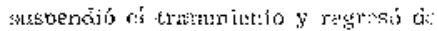

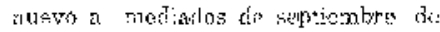
1.361 otriz vaz con roricilel'a y loz sece.

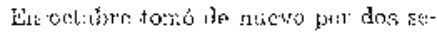

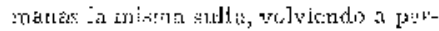

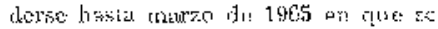
prendatif con rechudecucia de los

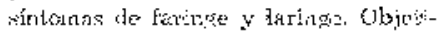

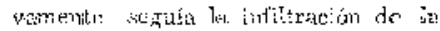

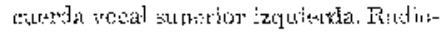

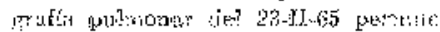

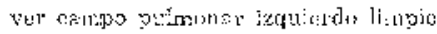

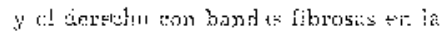

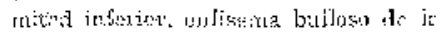

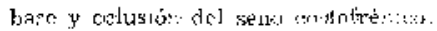
Fiste visperifo rasioliogico no se ba ne-

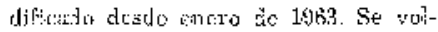

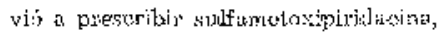
sin que haye despues resgresado para

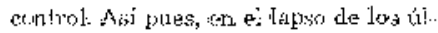
timos nutyr irleses retribir solatietale \& sen?alitis de triatuuijento rspenifjeo, ade. más rlel que tuvo por un mes en 1961 sifmpte de manera isceguler e incom-

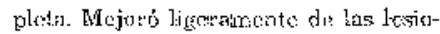
rus de la larimge.

\section{RESUMPN}

Puciente de 48 años que residió on var:ias provineisa de la Costat eon gin. givitis $y$ adentis cerrical que sulría perítus de mejoría y agravamiento e infiltersudo radiológico dusarte tre;

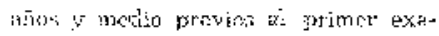

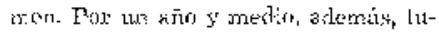

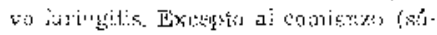

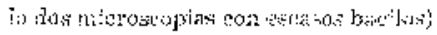

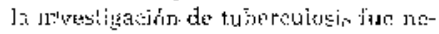

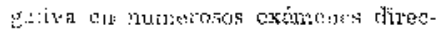
lo: $\forall$ por eultive Haliage de $\mathbf{l}$, bra-

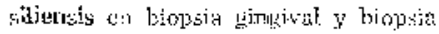

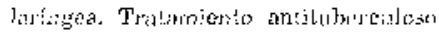

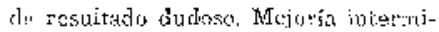

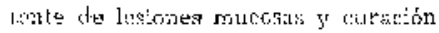
dl:! proneso fultmonas tou silifas que reaib:ó de manera irressular e juenon-

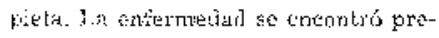

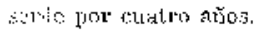

CA:30 Ne 4. JS.C. (Historis 1:5781). De 18 años de elad, func:onatus Mujucipel sos tosiden.te en Rhacis:

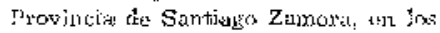

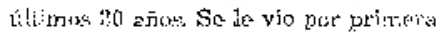

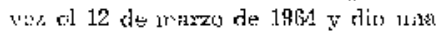
histulia de tres moses de dismen frovgresiva ctue se habis. hecho finalmontic

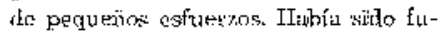

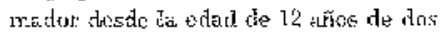

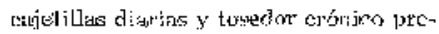

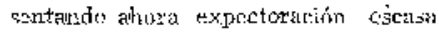
de elolor verdosu. Por ei znisino purio.do te tiowipo tuve natestar generial: sudkr, astenia, anorexia y habíu pis.. d.ro unas cinco bibrus the peso.

Ein noviembne? de 1922 sufrió traumatismo en el testículo vieresho es] cual. se hinchó y so puso dillorroso. tin un examen médico se excontró ́tumicración del nceticulo, ndwa y sertsible it? tacto. En le misma época, en radiografía pulmonar se vin pocos nódútos (unos doce) en flas zonas superiores 


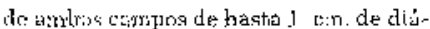
Inetro, de eccasa dernidad $y$ de crontor-

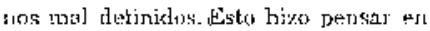

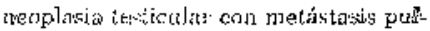

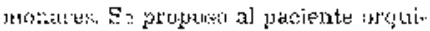

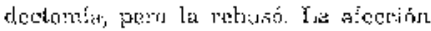

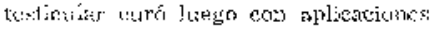

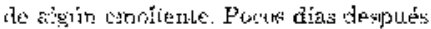
de la astmisirss, aztes fo cononzado ol

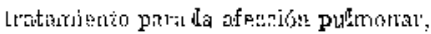

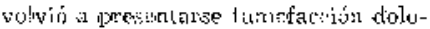
trua dei it stículu delecho, pero asimis-

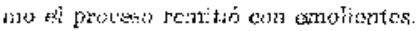

En exann!n físiro se tenía un cul1truo delgarlo peio no maciado con po-

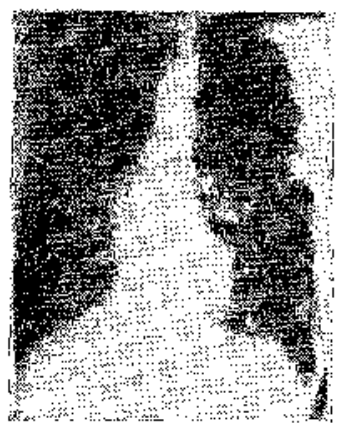

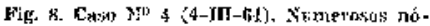
dulos isreguiturs y mal aletinidos de 1 a $3 \mathrm{~cm}$.

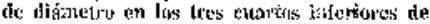
aulsos julmones.

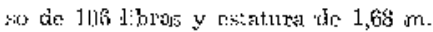

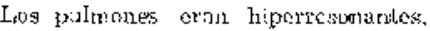

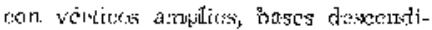
dirs y desoparesda th matiles prectsdiex]. So ausxultabil rewles húmexins fin

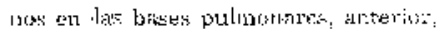

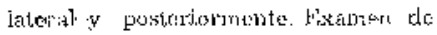

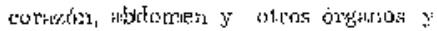

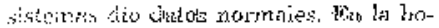
cat hubo ud dencia tol:a] y rir sie vrjan le-

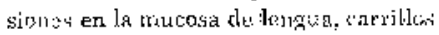

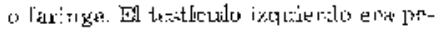

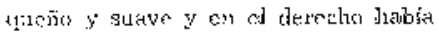

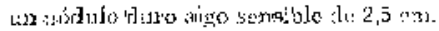
miln:rav ius epididimos y cestetorts: se

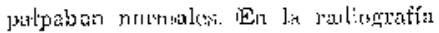

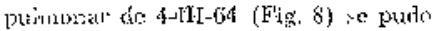

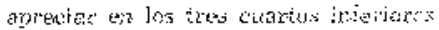
de anbor campos numerosus ródilux de conternos irregtiares y mal defir:dos te tno it tios cm. Le ciánetro. Los

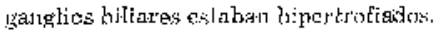

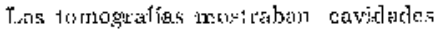

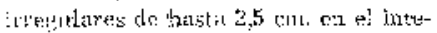

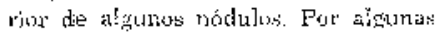
placas radiográficus que existian dos-

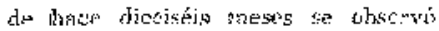
cure las modulos iban aumentando s::

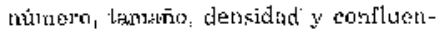

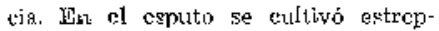
tococo viridans. Un cxamen citslú(j) con colmación japanicolnou fue negaljvo. Fil eximen microsópicu fuc negetivo para ácido-resistentes; $y$ uo eut tivo negat:vo paria hacilo tuhernulcyr. Un cultivo para hongos patógenris dio resultarlo neyrativo. En existucn en frescu tampoco se encontró jyetrisitcs. Is orimin no presentó elementos anormetleg. Fn lit sangre hutbr 4:"600.800

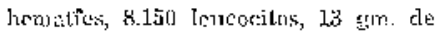
hemogrobina, $17 \%$ de seggrnentades

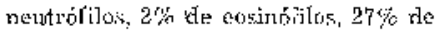
linfocitons, $40 \%$ do thematoestu y 29 min. de eritrosedinnntaciún. Lil reist: :́n de Manloux fuc positiva (10 mm), 


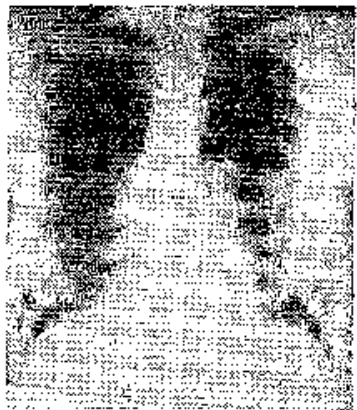

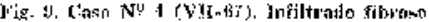
difuso de Jas cegiontex inferiores de atobos mulinonesi.

A wartir de los últimos diés de sbrill

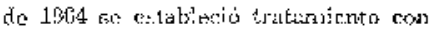
iriple sulfar de dosir de 2 gin. al tía que

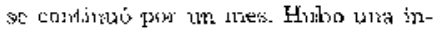
terrupcín do tiu ares y se reinció con 500 sus. por día de sullimetoxipiridal cinn- sulfanutiacina, medisación qut be mankivo por sieute rieses mís de ma* reri ilintermusupikla. Al. edbo de este tirstups a] esiads general ero bueno, hasía aumentatelo 38 librus de peso, no tenía sinten! ins ni sisnos respiratorios. Trumpuco hùbian reseurvid düs molestias de los lestítulos y se encontró el izquierritn peifunono y suave y el derecho

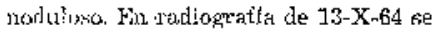
aprectabun lesinnes fibrosas difusas en atrbas mitades inforiores de los pulmoues que pertintueteron estabilizadas husti V11-67 (Fig. 9) m que cl enfermo cant.ju(dab) asinimnático por parto diz aparato resprintatuju.

\section{RESEMTFN}

Linfermo di: 48 diñs protedente de

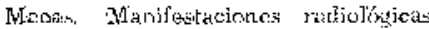
[n]lionatres progreritivas por 16 meses $y$ sir tonas respiratoriks y gonerales, cre cirntes grox tres moses, atemés de in-

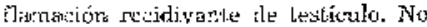
hiaho iesiones terumentaras. St? descattó tubereulouiz, aunque jo se de-

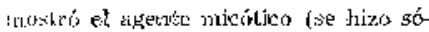
to urs examen directo y un cultivo de explato). 'La enferymedad curó con tratai. viocto de coho meses de nullas, 1o que kace pulwar on la pospbilidat de blas tonicosis sudamericana, pero también pricric liratartie de cotris micosis más raras romo nocartinsis, axtinumicosis a madiscomitusis que también son sensibjes âl rusticanento (1).

\section{DISCUSHON}

Fin primer halliazgo de Pexacoccidioides brasiliensis en cl Ecuador to hizo Mort'alván en 1948 (2) cu un tasn del Dr. Juan F. Invinert. Alvarez y Leone (3), Freire Potes (4), Rizzo y Arzube (5), Ortuga y Arguirallo (6); informan sorbre senctos easos de blastomicosis sudamericana pulmonar. acompantada, en ianar a otra vez, de lesiones de la piol, mulosa bracal y laringe, Fis un simpor sio sobre micasis profundas que tavo Jugar en 1960 on el Instituto Nacional do Higriente (7), Briones presentó un pikiente cor nubueulosis pulmonar $y$ paracarcidioidonicosis de lat laringe y Durand Nicola otros das cm afersion yaztmonar en quienes no sxi cncuntró bacilo tuberculosas pero si el 
hungs (:allwal. Zeregd (8), e:c importante material de recropsia, hajló del enformedud un sinco paciontes que torian lesiones to pulimunes, gangisos lincíticus, medtastinales $\mathrm{y}$ mesentéri-

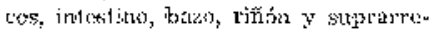

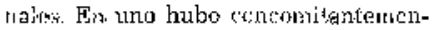
te tuluersulıxis puhmonar. Máronez, Cortader y Serrano (9), heldetarn c!l Cuenca nus yaciente con alectión pmlxnunar y culancomucosa y jlescruben lat

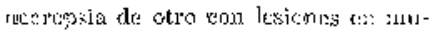

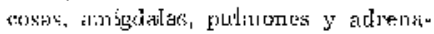
lne. Rodrínicz (10) en sus extensos

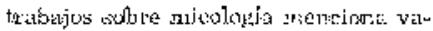
Jits entifermas con puratorcidiviramicalsis pulmomitr. En resutnpur, en el' lapso

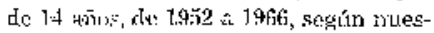

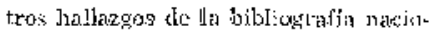

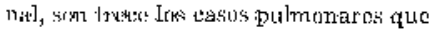
se has publicado con demostración del

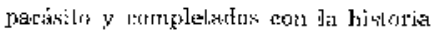
clínica o con la necropsia.

Los vascis repoltiatus en ell p.lesente tru.bujo snu thoumbes the 38 a 48 añs de adad que veriun cle zomas cálidas y lúainedes. Ningeno fue autúctono des la Sícrara. En los tres se ecmprobo la et:olusia y wno soliamente os suspechoso, aungles en íl los oxátrienes do labntatorio no se repitiven eomo hubiera toim do ennumenien'te.

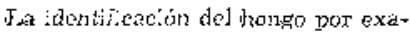
uncu dicento, pror cultivo o pur histopatología ej i manera de cstablecer el

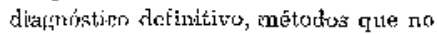
siempre nos lestularun fáciles por falita te medine, como so ilustra por li serio de criblidios que hubo que efeciual en cll caso N' 1. Por la misma razón, no se pudo accurrir a otros procedimien-

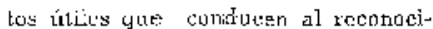

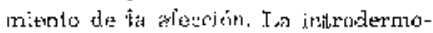

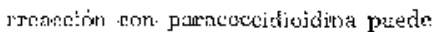
diur jalsás retatecinnes positivas y falsas

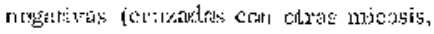

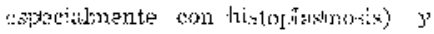

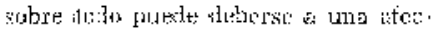

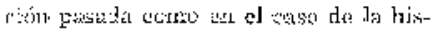

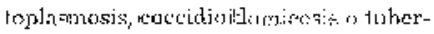

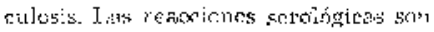

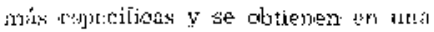
alk proporción dte en[errutos $(7,11)$.

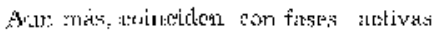

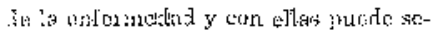

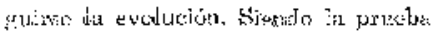
do jets:ipilas:ine de técrica más sensi-

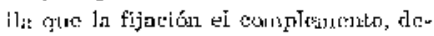

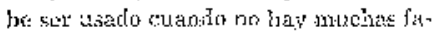

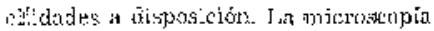
Clourascentle tumbitér ofuece posilvilidadcs paria ésta $y$ cotras enteromidudes a homgridi (12).

La búsquata rejturetla del bartite tu-

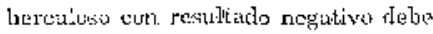

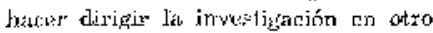
sontitlo, sobre toubo si se trata de entesmos gut: liemen caveruas $y$ due han sido sornctidos, sin xesullado, al trataunicrio autituberculnso. Fintonces debe

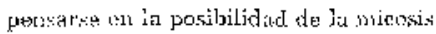
de que se visur thablando o de otra de difpiente nél.uralene.

Nuestros crafumes se presentarn con años o meses de sítulomas de crifermedid rénicu. En los tres de blantomicoijs se jnixió con las manifestaximnes dos lev buca o faringse que posiblemente procedieror a la enfermedad palmumax y esta ascuciateión bien conocida es ienporiante para orientar al dirnnóstico de wna neumzoputia que fácimento se 


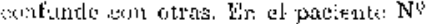

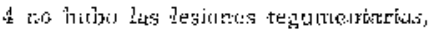
hechri que ya tra sidu señedado en la h'astchtiviss:s sulturrericana $(13,14)$. Sin embargo, os posibie que on algim momeño de da evolutión de de afec-

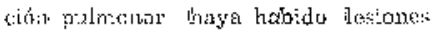

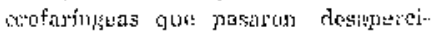

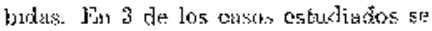

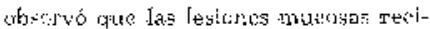

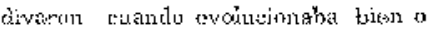
curat:a el en:mpromiso pulonfmar. Rudrifgucz y Rincén, sitados por Borreru

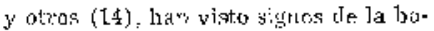
on trasta seis messes tespués del diac-

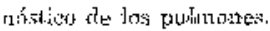

Por nuestras observecisones y por

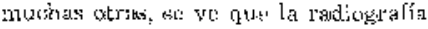
pulmoner so tiecue nadu do cacaclerístiva. Cor fuecuerula se aseaneja a la de la tuberenjosis. A presar de clln putede

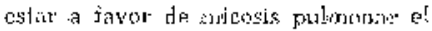

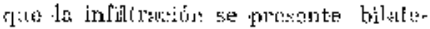
vilumestes con disposisión śmélstica $y$ proforcntemente st los totsios medior

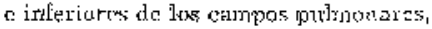
con bonderici'd a . Ta formacion de múltiples cevidadres por abseesos. I.0s casos prentustadosi tesían estos elencenLus de presunción. Tatrifín en do de otutis nicosis quy themos publ:cado, uno te geofricosis (15) y ottis de uriptocostatis $(d b)$, st: baliló una imagen rartiológis:a pelrecict. ifstos no quere descir cite no baysul cuadros que:

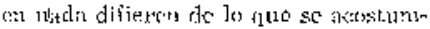
bra a ver en condiciums patolúgicas de

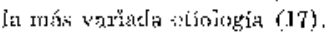

4: Inatatuiculo de los pacientes de paruecevidioirmatukits sc hize con sulla: เuyo resullado ba sido claro e in* nedialo solane lias diferentes loceliza.

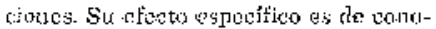

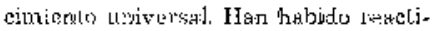
vactones ruarddo ]: Inedlicactón ho se

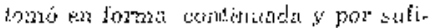

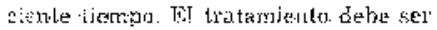
itolingad\%, flieunes cren cue debe

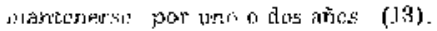
Cuande n no bier trileralo pliede

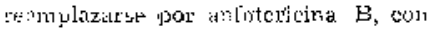

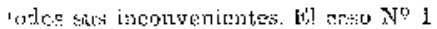

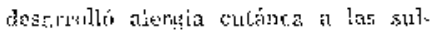

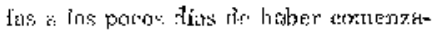
de: peros no rcapiesedi desplués df pocos ditas to statstrión $\mathrm{y}$ reiniciación cors ir. mismus atroya.

\section{IESLMLEN}

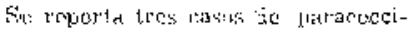

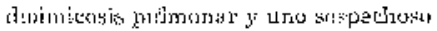
(1) of que hubo oxalisenes insuficientas pero rqur curó ion la rme clickción espećfiva. Curresponders a bonkres de 38 a 48 itris de exhad, procesäentes de zoJras rílidas y hú:medas, singunn autócto: de da lasicrra. Fl diagnóstico de esta mick mis poofuruda se hàce pror la de. nosilsación thel agerte sutsal que is noceswio buscurlo refiloratanente 'en sereciones bromulates $y$ bi lujidos. Yä concernituncia $\mathrm{H}_{\mathrm{i}}$ Jesionos mukucutíngas es frecuence y so eventiv 1. ${ }^{\prime}$ en tres enfexmo: Ciertis caracteristicss radiológicas puteder servir de gula rn el diagnóstico de micosis pulmown: y tonsisten cri infiltracioncs bilixterales, s:métricus y con tendencia th Fluscco'ersen. El tratamiento se hizo con

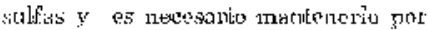


Iorgo dempo paya evitur recidivas. Se lrece una revisción de Ia bibioggraila n'scional en la cue se ba encuntrado gros 13 casus deste 1952 hisila 1066.

\section{SUMMARY}

Three cates of ptulmonary parteosetdioidomicosis we reporled. Oxe more is susper:ted in wirth laburatury exa. minations were insuffirient. but cured with sperilis therapy. Thoy ware men betwecn the ages of 33 to 48 from warid ibumild zones, none from the Sio rra (highlands). The diagnowis of this deep moveoses is made by renograntion of the nyemt in themehial steretions or bicksy of disnased tistlues and must be repeatceliy soushbt. Nuco and cuteneous lesions are freciuent and ware prescrt in threc patikents. Some ridt:ological patitem may be a gu!de for diagrosis of plillnonary myeoses, which consists in bilatedal symctric infiltrates will tenderity to auscess formution. Trcirtuneril was done with sulfa chus and it: mist pe maintained for is loolng periokl of $1 \mathrm{~m}$ e to avoid tecurromec. In revietvilus the bibliograplyy, 13 edses hitwe been rosported in the country lirotic 1952 lo 1966.

\section{BIBLFOGRAFIA}

\pm Comnathe on Thesaps, Mmesibar Tho-

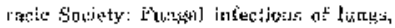
Am. Rev. Ecs!l. Dis., 8ĩ: 784, 133.

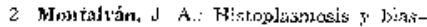

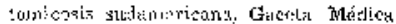
3: $2015,254$.

4 lizeja: Poles, A.: Cuntr!luaión al esiudion

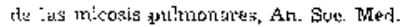
Quit. Guayus, 4i, Ne 4-7: 2530, 1954 .

5 Rizzo, Ii, y Arrulbr, MI.: Plestoulicosis

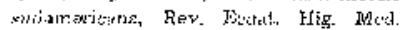
l'rop. 14: 7, 1957.

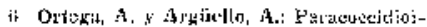

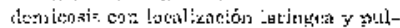

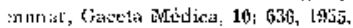

$\bar{i}$ simpäis solure miensis mofundas: Ruv.

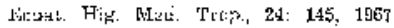

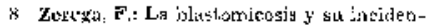

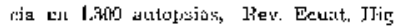

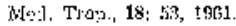

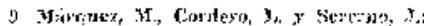
J3listomicesis snelarreritina, Rev, Fác.

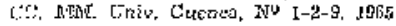

10 Inotriguez, J. D.: A propósitu de un easn de blastonicosjs sudarericana pronerlente

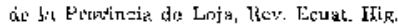

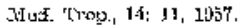

11 Restzenn, A,: Comportioniento inmutuolín furu de vinte pucientes cor paracocei-

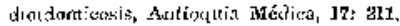
J\$SI.

I2 Chtek, k. W. Tistrescense mioroscupy for

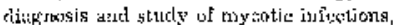
Am. Kev. Resp. Lis., 92 (Pa:L 2): 175, $1 ! 65$.

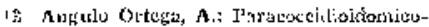

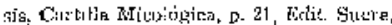
Cun'ucas, 1959 .

14 Forrevo, J., Resirono, A, y lobbleto, HI.:

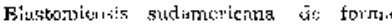

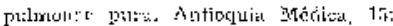
Si13, ISFFr.

1.̄ Trivalos, R. y alcus, ha: Geotricosis yulmona:, Gilet!ia अedink, 9: 15j, 1954.

1i) Arress, L. Y hltegfia, A. Criptosecosus pul-

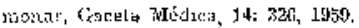

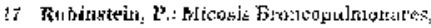

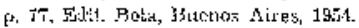

